\title{
FABRICATION AND MEASUREMENT OF EFFICIENT, ROBUST CESIATED DISPENSER PHOTOCATHODES*
}

\author{
E. J. Montgomery", D. W. Feldman, P. G. O’Shea, Z. Pan, U of MD, College Park, MD 20742 \\ N. A. Moody, Los Alamos National Laboratory, Los Alamos, NM 87545 \\ K. L. Jensen, Naval Research Laboratory, Washington DC 20375
}

\section{Abstract}

Photocathodes for high power free electron lasers face significant engineering and physics challenges in the quest for efficient, robust, long-lived, prompt laserswitched operation. The most efficient semiconductor photocathodes, notably those responsive to visible wavelengths, suffer from poor lifetime due to surface layer degradation, contamination, and desorption. Using a novel dispenser photocathode design, rejuvenation of cesiated surface layers in situ is investigated for semiconductor coatings building on previous results for cesiated metals. Cesium from a sub-surface reservoir diffuses to the surface through a microscopically porous, sintered tungsten matrix to repair the degraded surface layer. The goal of this research is to engineer and demonstrate efficient, robust, long-lived regenerable photocathodes in support of predictive photocathode modeling efforts and suitable for photoinjection applications.

\section{INTRODUCTION}

\section{Photocathodes for FELs}

High power free electron lasers (FELs) place unique, stringent requirements on the cathode because of constraints on the beam itself (current density, emittance, temporal structure, etc.) [1,2]. For a representative RF accelerator operating at a few tens of $\mathrm{GHz}$, an electron bunch just a few ps long is needed; therefore one must have a prompt switching response, for which the laserswitched photocathode is the preferred emitter. As shown in Figure 1, a pulsed drive laser causes electron pulses to be emitted from the cathode in synchrony with an

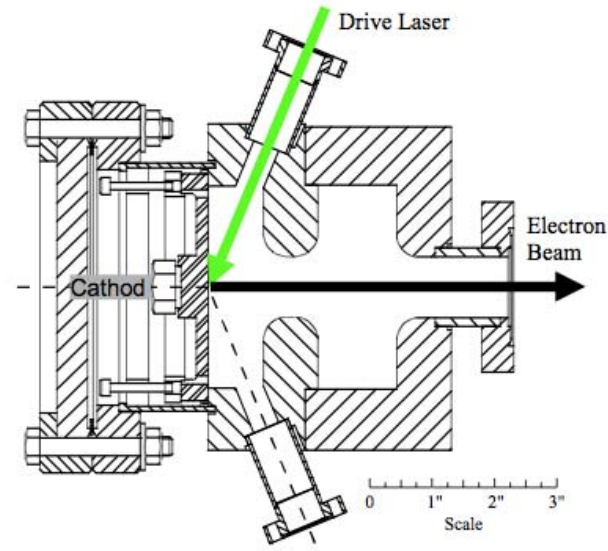

Figure 1: Photoinjector schematic accelerating RF field. An important characteristic of photocathodes is quantum efficiency $(\mathrm{QE})$, the ratio of emitted electrons to incident photons. QE varies with wavelength, and longer wavelength drive lasers are advisable based on laser power stability considerations in second harmonic generation (i.e. frequency doubled YAG/YLF lasers); however, high current density and laser heating considerations lead to requisite QEs of a few percent at the drive laser wavelength. High QE and long lifetime are, unfortunately, competing parameters for photocathodes. The highest QE photocathode materials in the visible spectrum, the alkali antimonides, typically last only a few hours or days before they must be rejuvenated by recoating the surface with Cs [3].

\section{Dispenser Photocathodes}

Dispenser photocathodes allow rejuvenation in situ, avoiding the need for complex and time-consuming refurbishment in a dedicated preparation chamber. The established technology of thermionic dispenser cathodes has been adapted, in the University of Maryland (UMD) design, to dispense $\mathrm{Cs}$ rather than $\mathrm{Ba}$ and to operate at temperatures around $120^{\circ} \mathrm{C}$. The UMD dispenser cathode, as shown in Figure 2, comprises a sub-surface reservoir and a diffusion barrier (in this case, sintered tungsten) which doubles as the photoemissive surface.

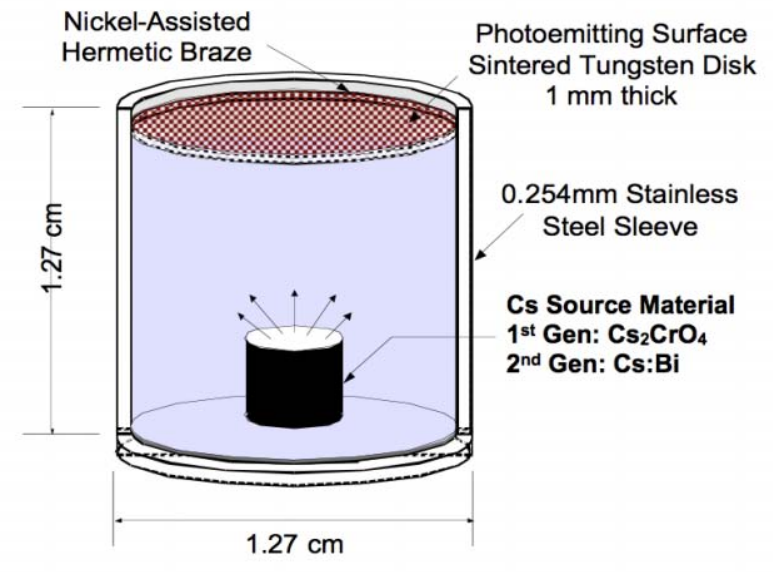

Figure 2: Dispenser Photocathode

\footnotetext{
*Work supported by Joint Technology Office (JTO) and Office of Naval Research (ONR)

"ejm1@umd.edu
} 


\section{EXPERIMENT}

\section{Apparatus}

Semiconductor layer depositions (for high QE cathode fabrication), cesium depositions, and quantum efficiency measurements are made using the apparatus shown in Figure 3. The UHV chamber is a six-way cross. The

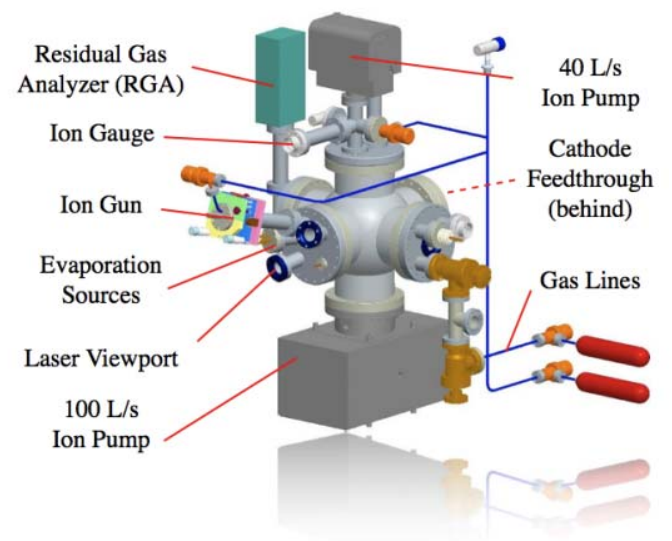

Figure 3: Experimental apparatus

cathode/anode assembly and cathode heater are on the back port, while the evaporative sources of alkali metals and antimony, along with the lasers and ion cleaning gun, point at the cathode through the annular anode from the front port. (The ion cleaning gun is a critical part of the apparatus, delivering measured doses of $6.4 \mathrm{keV}$ Ar ions to sputter contaminants off the surface of the cathode, leaving an atomically clean substrate for later tests.) Top, bottom, and side ports allow pumping, pressure measurement, and residual gas analysis. Five laser wavelengths $(375,405,532,655,808 \mathrm{~nm})$ cover the visible spectrum and permit investigation of wavelength dependent parameters and determination of workfunction. All signals (pressure, temperature, photocurrent, anodecathode voltage, evaporated layer thickness, and background gas composition) are monitored by LabVIEW, which also controls the evaporative sources and automatically moves the lasers to illuminate the cathode in turn while measuring photocurrent and calculating QE. A QE calculation consists of a low-noise photocurrent measurement, at a given wavelength and stable, known $\mathrm{CW}$ diode laser power, used in the formula

$$
i(\mathrm{~mA}) \approx \frac{\lambda(\mathrm{nm})}{124} \bullet P_{\text {laser }}(\mathrm{W}) \bullet Q E(\%)
$$

QE can be monitored as a function of time while surface conditions are systematically varied (coverage or layer thickness, temperature, gas composition, etc.).

\section{Cesiated Metals}

We have conducted a detailed investigation of cesiated metals (specifically the Cs-W system) [4]. To summarize these results, which form the foundation of our current research: first, we have developed in collaboration with NRL a theory of quantum efficiency of cesiated metals as a function of surface coverage of cesium; theoretical predictions agree well with experiment without the use of adjustable parameters, as shown in Figure 4 [5]. It is important to note that this theory has recently been updated, and now accounts for electron energy dependence of thermal transport and emission probability by relating those processes to fundamental scattering models, and also now includes a Moments-based approach which improves the theory for photon energies significantly higher than the work function.

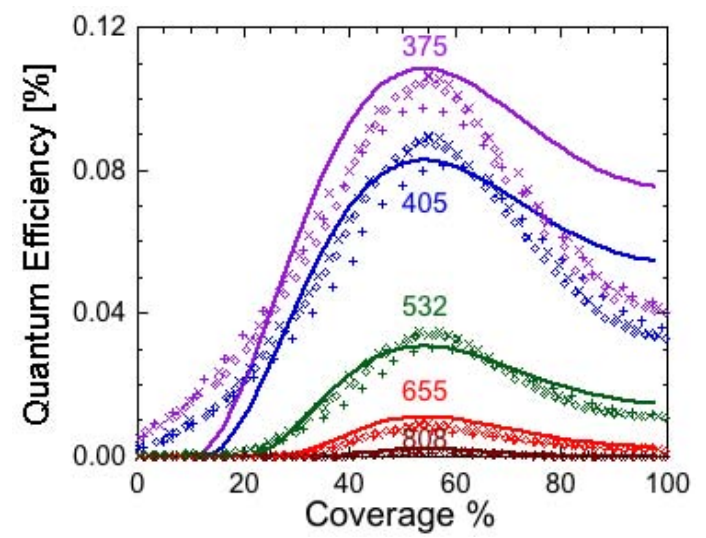

Figure 4: Cs-W QE vs coverage (\% of one monolayer), updated theory (solid lines) and experiment (symbols)

Where discrepancies between theory and experiment are apparent, it must be remembered that the real surface is not perfectly free of contaminants, nor is it smooth, as the theory assumes. To continue with the summary of our cesiated metals research: second, we have demonstrated successful operation of a first-generation dispenser photocathode (using a cesium chromate-titanium reservoir material which reduces to elemental cesium at $475^{\circ} \mathrm{C}$ during initial activation) with an extended lifetime under continuous rejuvenation of 47 days at $160-180^{\circ} \mathrm{C}$, compared to 5 days at $25^{\circ} \mathrm{C}$ for an unrejuvenated Cs-W cathode. And third, we have designed and demonstrated a second-generation dispenser photocathode using a Cs: $\mathrm{Bi}$ intermetallic compound as the reservoir material, which offers lower activation and operation temperatures $\left(270^{\circ}\right.$ and $120^{\circ} \mathrm{C}$, respectively). Lower temperature operation will be important for successful rejuvenation of temperature-sensitive high-QE coatings in the future. The second-generation cathode also uses a thinner sintered tungsten diffusion barrier ( 20 mils vs 40 previously) to enhance cesium diffusion to the surface at lower temperatures.

\section{Alkali Antimonides}

Our current research focuses on fabrication of alkali antimonide coatings to achieve high $\mathrm{QE}$, and how to best rejuvenate such coatings using the dispenser photocathode. We have completed tests of the simplest cesium-based antimonide, $\mathrm{Cs}_{3} \mathrm{Sb}$. Fabrication of cesium antimonide consists of an initial layer of antimony, heated 
to $130^{\circ} \mathrm{C}$, at which time cesium is evaporated onto the surface. As QE levels off after its initial rise, the cathode is cooled while maintaining a deposition rate of $\mathrm{Cs}$ of approximately $0.1 \AA / \mathrm{s}$. We have achieved quantum efficiencies of $11 \%$ at $375 \mathrm{~nm}$ and $3.3 \%$ at $532 \mathrm{~nm}$ for an initial $100 \AA$ thick antimony layer, as shown in Figure 5 .

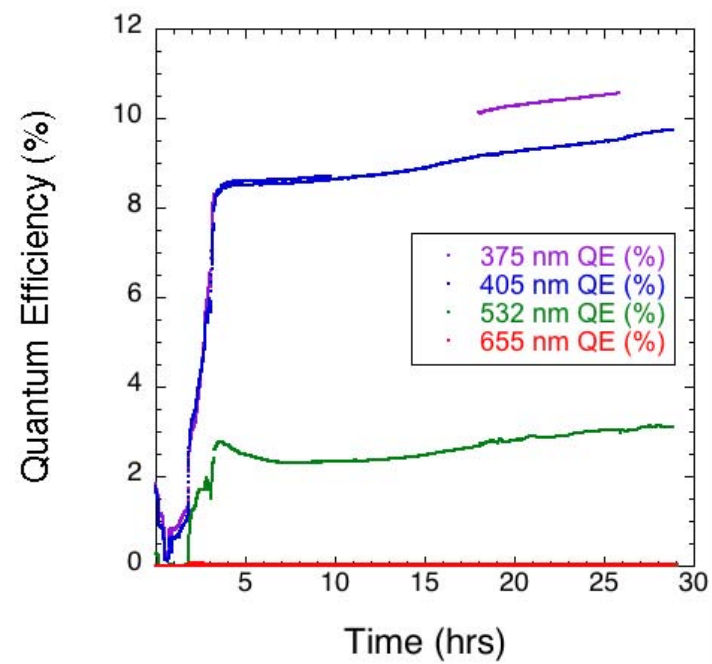

Figure 5: Fabrication and demonstration of high QE using the $\mathrm{Cs}_{3} \mathrm{Sb}$ semiconductor

The break in the UV laser data is due to a gap in data collection, not a physical effect.

Results from temperature variation tests indicate that temperatures below $50^{\circ} \mathrm{C}$ do not adversely affect the Cs$\mathrm{Sb}$ layer (QE returns to nominal after cooling back to room temperature), but temperatures above $50^{\circ} \mathrm{C}$ result in QE degradation even after cooling back to room temperature, most likely due to loss of Cs in the surface coating. Although most $\mathrm{Cs}-\mathrm{Sb}$ cathodes are fabricated at temperatures well above $50^{\circ} \mathrm{C}$, they are fabricated using external evaporation sources which allow a slow but continuing evaporation of $\mathrm{Cs}$ onto the surface as the cathode cools below $50^{\circ} \mathrm{C}$. The dispenser concept does not allow a similar continuing Cs supply because the minimum diffusion temperature for our dispensed $\mathrm{Cs}$ is above $100^{\circ} \mathrm{C}$.

For this reason we are in the process of moving to potassium cesium antimonide, $\mathrm{K}_{2} \mathrm{CsSb}$, as a candidate material for a high QE rejuvenable dispenser photocathode. With a more temperature stable photoemissive material, it will be possible to match the loss of cesium at the surface (due to evaporation or ion back-bombardment) with the diffusion rate from the reservoir beneath the surface, achieving a balance between cesium arriving at and leaving the surface.

Experimental results continue to be applicable to the development of a predictive semiconductor photoemission theory. Through our ongoing collaboration with NRL, a photoemission theory for systems like the alkali antimonides is being investigated.

\section{CONCLUSION}

\section{Future Work}

- Investigate high QE alkali antimonide coatings compatible with dispenser operation and cesium diffusion temperatures

- Continue developing predictive photoemission theories in parallel with experiment

- $\quad$ Predict optimum pore size and pore spacing using a cesium diffusion theory

- Design and construct a dispenser cell with controlled porosity following the optimum parameters predicted by theory

- Test prototype dispenser in a working RF gun following successful demonstration at UMD

\section{Summary}

Photocathodes for free electron lasers face competing requirements of high $\mathrm{QE}$ and long lifetime. The dispenser photocathode is being investigated at UMD as photoemitter which will be rejuvenable in situ. Initial tests focused on the cesiated tungsten system. Agreement with a new theory for the QE of cesiated metals as a function of coverage was good. The present apparatus allows the fabrication of alkali antimonides; cesium antimonide was successfully fabricated on a dispenser using external deposition. Due to temperature sensitivity of the material, we believe a more promising candidate for a rejuvenable high QE semiconductor layer is potassium cesium antimonide, and work is progressing to demonstrate and probe the bialkali system. A high QE, robust dispenser will help enable photocathodes to meet the requisite standards demanded by high power FELs.

\section{REFERENCES}

[1] P. G. O'Shea, H. G. Freund, Science 292, 1853 (2001).

[2] S. H. Kong, J. Kinross-Wright, D. C. Nguyen, R. L. Sheffield, Nucl. Instrum. Methods Phys. Res. A 358, 272 (1995).

[3] A. H. Sommer, Photoemissive Materials (Wiley, New York, 1969).

[4] N. A. Moody, K. L. Jensen, D. W. Feldman, P. G. O'Shea, E. J. Montgomery, Appl. Phys. Lett. 90, 114108 (2007).

[5] K. L. Jensen, Development of a General Formulation of Thermal, Field, and Photo-induced Electron Emission. J. Appl. Phys., (2007). Submitted for publication. 